\title{
Validation of a Harmonic Model for Eddy Currents in Slitted Conducting Plates
}

\author{
C.H.H.M. Custers ${ }^{* a)}$ Non-member, J.W. Jansen* ${ }^{*}$ Non-member \\ E.A. Lomonova* Non-member
}

(Manuscript received Oct. 23, 2017, revised Dec. 18, 2017)

\begin{abstract}
The paper describes a 3D semi-analytical harmonic modeling technique that is capable of modeling eddy current distributions in segmented conducting structures, such as slitted conducting plates, and the associated magnetic fields. The spatially varying conductivity of a conducting region is incorporated into the solutions of magnetic-field quantities and the induced current density. The harmonic model is compared to results obtained with finite element analysis. An experimental setup is used to measure the field distribution above differently slitted conducting plates, in which eddy currents are induced by a coil. The measurement results are compared to simulation results, and the perturbations are analyzed.
\end{abstract}

Keywords: analytical modeling, Eddy current, Fourier analysis, permanent magnet machines, finite element method, experimental validation

\section{Introduction}

Accurate prediction of eddy current distributions in electromagnetic devices is important for optimization of the efficiency and performance of applications with high frequent ac magnetic fields. Eddy currents cause power losses in (highspeed) electrical machines ${ }^{(1)-(4)}$, and can reduce the performance in wireless power transfer devices ${ }^{(5)}$. In electrical machines, eddy currents are induced in conducting parts, such as magnets, cooling systems and construction materials, due to movement and ac magnetic fields. In nano-meter accurate positioning devices, used in e.g. the semi-conductor manufacturing industry, such as planar motors ${ }^{(6)}$ and core-less linear motors, the parasitic force due to the eddy currents can reduce the performance ${ }^{(7)(8)}$. Analysis of the transient eddy current distribution can give important information on the spatial and time dependent harmonic content, which can be used to increase the performance of the machines.

To calculate these eddy currents and parasitics created by them, the finite element method (FEM) is an often used method ${ }^{(9)}$. To accurately model eddy current effects for motors such as the linear and planar motor mentioned before, the geometrical shape of the conducting material has to be taken into account in all three dimensions. However, the large open boundary structures of these type of motors makes the 3D FEM have a relatively high computational load. For this reason, a semi-analytical harmonic (Fourier solutions based) method $^{(10)(11)}$ has been researched as an alternative for the FE method. The harmonic model that will be described and validated, is capable, in contrast to classical harmonic models,

\footnotetext{
a) Correspondence to: C.H.H.M. Custers. E-mail: c.h.h.m.custers @ tue.nl

${ }^{*}$ Electromechanics and Power Electronics, Eindhoven University of Technology

5600 MB Eindhoven, The Netherlands
}

of calculating eddy currents in $3 \mathrm{D}$ complex segmented conducting structures as was described in ${ }^{(12)}$ for $2 \mathrm{D}$ problems.

In this paper, a semi-analytical method to model eddy currents in slitted conducting plates is presented. This method can be used to analyze eddy currents in the cooling system of a moving-magnet planar motor. As this paper focuses on the validation of the semi-analytical method using the finite element method (FEM) and measurements, an electromagnetic configuration is used that only consists of a single coil and a copper plate. The configuration will be described first. Then the (Fourier based) model formulation will be discussed for conducting regions. To verify the developed model it will be compared to FEM results and measurements.

\section{Electromagnetic Configuration}

The electromagnetic configuration to which the harmonic model is applied and which will be used for verification is shown in Fig. 1. A race-track shaped coil is placed in air with on top a rectangular copper plate. A $50 \mu \mathrm{m}$ high airgap is present between the coil and the plate. An ac current in the coil will produce a time varying magnetic field which induces (eddy) currents in the copper plate. In total, three rectangular plates, depicted in Fig. 2 and numbered for the remainder of the paper, are analyzed. The first plate does not contain any slits. The two other rectangular plates ( 2 and 3 ), contain a different slit pattern which will change the eddy current distribution. The slit pattern in plate 2 has been chosen such that the spatial frequency of the slits in the $x$-direction is higher than in the $y$-direction. In plate 3 the opposite is done. In this way, the effect of the spatial frequency of the slits in relation to the number of harmonics in both directions is investigated. Moreover, the patterns of the slits are typical for cooling plates in linear and planar motors.

Because a harmonic (Fourier based) modeling technique is used to calculate the magnetic fields and eddy currents, 


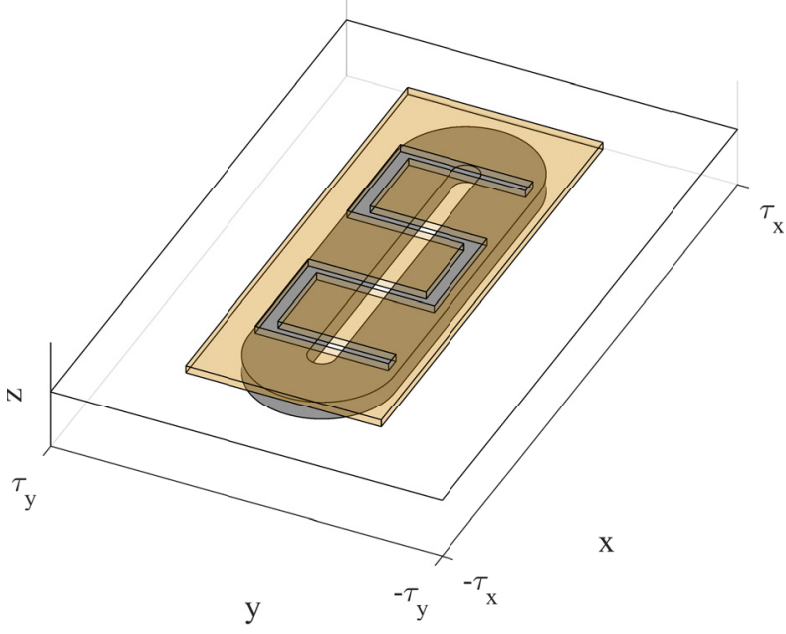

Fig. 1. Electromagnetic configuration with a slitted plate used for verification

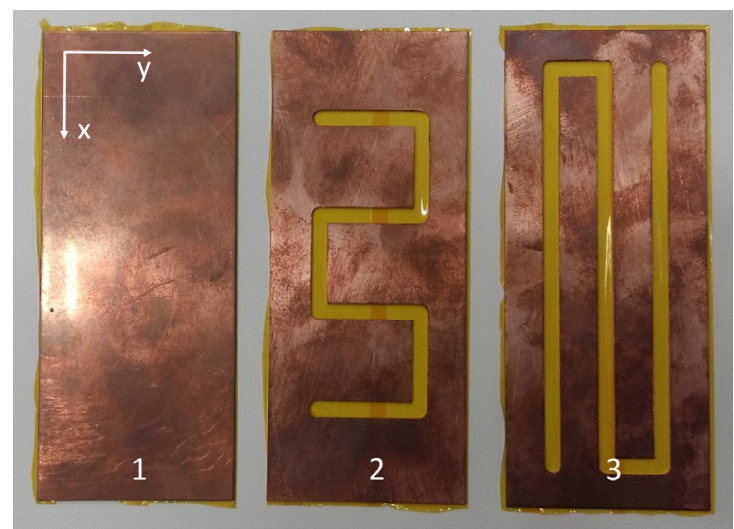

Fig. 2. The three copper plates that are studied in this research. The bottom side of the plates is covered with polyimide film for isolation purposes

the geometric model of the electromagnetic configuration of Fig. 1 is assumed to be periodical in both the $x$ - and $y$ directions. However, as the measurement set-up will on consist of a single 'period', and is not repeating in the $x$ - or $y$ direction, air is added on both sides of the periodical section. The periodic width in both directions is almost double of the coil width (see Table 1) in that direction. In this way, the influence of the magnetic fields of adjacent periods is minimized. In the $z$-direction, the model is divided into regions. The (Fourier based) formulation of the magnetic fields in the non-conducting regions is described in ${ }^{(13)}$. In the conducting region, where the copper plate is located, the conductivity will vary as a function of position. Hence, outside the plate and inside the slits (where air is present) the conductivity is zero. In the next section, the formulation for this region will be presented.

\section{Harmonic Model Formulation in Conducting Regions}

The magnetic field solutions are based on the quasi-static Maxwell's equations. This means the displacement current, $\frac{\partial \vec{D}}{\partial t}$, is neglected. The time derivative is denoted by $j \omega$. Maxwell's equations are then given by
Table 1. Model and experimental setup properties

\begin{tabular}{l|l|l} 
Description & Value & Unit \\
\hline \hline Half periodic width in $x$ direction $\left(\tau_{x}\right)$ & 80 & $\mathrm{~mm}$ \\
Half periodic width in $y$ direction $\left(\tau_{y}\right)$ & 50 & $\mathrm{~mm}$ \\
\hline Coil length (in $x$ direction) & 107.8 & $\mathrm{~mm}$ \\
Coil width (in $y$ direction) & 37.0 & $\mathrm{~mm}$ \\
Coil height & 1.7 & $\mathrm{~mm}$ \\
Bundle width & 14.3 & $\mathrm{~mm}$ \\
Number of turns & 59.5 & - \\
Filling factor & 0.8 & - \\
\hline Plate length (in $x$ direction) & 120.0 & $\mathrm{~mm}$ \\
Plate width (in $y$ direction) & 50.0 & $\mathrm{~mm}$ \\
Plate thickness & 0.95 & $\mathrm{~mm}$ \\
Slit width & 4 & $\mathrm{~mm}$
\end{tabular}

$$
\begin{aligned}
& \nabla \times \vec{E}=-j \omega \vec{B}, \ldots \ldots \ldots \\
& \nabla \times \vec{H}=\vec{J}, \ldots \ldots \ldots \ldots \\
& \nabla \cdot \vec{B}=0, \ldots \ldots \ldots \ldots \ldots \\
& \nabla \cdot \vec{J}=0 \text { (no free charge), }
\end{aligned}
$$

where $\vec{E}$ is the electric field intensity, $\vec{B}$ the magnetic flux density, $\vec{H}$ the magnetic field strength, $\vec{J}$ is current density and $\rho$ is the electric charge density. The relation between the magnetic flux density and the magnetic field strength is given by the constitutive relation

$$
\vec{B}=\mu_{0} \mu_{r} \vec{H}
$$

where $\mu_{0}$ is the permeability of vacuum and $\mu_{r}$ is the relative permeability, which is equal to 1 throughout the model. The eddy current density $\vec{J}^{e d d y}$ is obtained from the electric field

$$
\vec{J}^{e d d y}=\sigma(x, y) \vec{E},
$$

where $\sigma(x, y)$ is the position dependent conductivity. After substitution of (5) and (6) into (1) and (2), equations (1) and (2) are written separately for each Cartesian component of $\vec{E}$ and $\vec{H}$. The expressions for the $z$-components are substituted in the remaining equations, resulting in

$$
\begin{aligned}
& \partial_{z} E_{x}=j \omega \mu_{0} H_{y}-\partial_{x}\left(\sigma(x, y)^{-1}\left(\partial_{x} H_{y}-\partial_{y} H_{x}\right)\right), \\
& \partial_{z} E_{y}=-j \omega \mu_{0} H_{x}-\partial_{y}\left(\sigma(x, y)^{-1}\left(\partial_{x} H_{y}-\partial_{y} H_{x}\right)\right), \\
& \partial_{z} H_{x}=-\sigma(x, y) E_{y}+\left(j \omega \mu_{0}\right)^{-1} \partial_{x}\left(\left(\partial_{x} E_{y}-\partial_{y} E_{x}\right)\right), \\
& \partial_{z} H_{y}=\sigma(x, y) E_{x}+\left(j \omega \mu_{0}\right)^{-1} \partial_{y}\left(\left(\partial_{x} E_{y}-\partial_{y} E_{x}\right)\right) .
\end{aligned}
$$

To find a solution to these four equations, the method of separation of variables is applied. As mentioned before, a harmonic basis for the solution in the $x$ - and $y$-directions is chosen. This means that every quantity, that is a function of $x$ and $y$, is written as a double truncated Fourier series

$$
f(x, y)=\sum_{m=-M / 2}^{M / 2} \sum_{n=-N / 2}^{N / 2} \mathbf{f}_{n, m} e^{j\left(k_{x, n} x+k_{y, m} y\right)}, \cdots \ldots \ldots
$$

where the spatial frequencies $k_{x, n}$ and $k_{y, m}$, are given by

$$
\begin{aligned}
& k_{x, n}=\frac{n \pi}{\tau_{x}}, \\
& k_{y, m}=\frac{m \pi}{\tau_{y}},
\end{aligned}
$$


where $\tau_{x}$ is half of the periodic width in the $x$-direction and $\tau_{y}$ is half of the periodic width in the $y$-direction. The coefficients of the double Fourier sum per harmonic pair $n, m$ are collected in a column vector denoted by $\mathbf{f}$. The Fourier series is truncated for implementation purposes and the number of harmonics used in the $x$ - and $y$-direction is equal to $N+1$ and $M+1$ respectively.

To obtain the solution in the $z$-direction, (7)-(10) are written in two matrix equations in the spectral domain,

$$
\begin{aligned}
& \frac{\partial}{\partial z}\left[\begin{array}{l}
\mathbf{e}_{\mathbf{x}} \\
\mathbf{e}_{\mathbf{y}}
\end{array}\right]=\mathbf{F}\left[\begin{array}{l}
\mathbf{h}_{\mathbf{x}} \\
\mathbf{h}_{\mathbf{y}}
\end{array}\right], \\
& \frac{\partial}{\partial z}\left[\begin{array}{l}
\mathbf{h}_{\mathbf{x}} \\
\mathbf{h}_{\mathbf{y}}
\end{array}\right]=\mathbf{G}\left[\begin{array}{l}
\mathbf{e}_{\mathbf{x}} \\
\mathbf{e}_{\mathbf{y}}
\end{array}\right],
\end{aligned}
$$

where,

$$
F=\left[\begin{array}{cc}
-\mathbf{K}_{\mathbf{x}} \boldsymbol{P}^{-1} \mathbf{K}_{\mathbf{y}} & j \omega \mu_{0} \mathbf{I}+\mathbf{K}_{\mathbf{x}} \boldsymbol{P}^{-1} \mathbf{K}_{\mathbf{x}} \\
-j \omega \mu_{0} \mathbf{I}-\mathbf{K}_{\mathbf{y}} \boldsymbol{P}^{-1} \mathbf{K}_{\mathbf{y}} & \mathbf{K}_{\mathbf{y}} \boldsymbol{P}^{-1} \mathbf{K}_{\mathbf{x}}
\end{array}\right],
$$

and

$$
G=\left[\begin{array}{cc}
\left(j \omega \mu_{0}\right)^{-1} \mathbf{K}_{\mathbf{x}} \mathbf{K}_{\mathbf{y}} & -\boldsymbol{P}-\left(j \omega \mu_{0}\right)^{-1} \mathbf{K}_{\mathbf{x}}{ }^{2} \\
\boldsymbol{P}+\left(j \omega \mu_{0}\right)^{-1} \mathbf{K}_{\mathbf{y}}{ }^{2} & -\left(j \omega \mu_{0}\right)^{-1} \mathbf{K}_{\mathbf{y}} \mathbf{K}_{\mathbf{x}}
\end{array}\right],
$$

and $\mathbf{e}_{\mathbf{x}}, \mathbf{e}_{\mathbf{y}}, \mathbf{h}_{\mathbf{x}}$ and $\mathbf{h}_{\mathbf{y}}$ are the vectors containing the Fourier coefficients of $E_{x}, E_{y}, H_{x}$ and $H_{y}$ respectively and $\mathbf{I}$ is the identity matrix. The matrices $\mathbf{K}_{\mathbf{x}}$ and $\mathbf{K}_{\mathbf{y}}$ represent the derivatives to $x$ and $y$ respectively and contain the spatial frequencies $k_{x, n}$ and $k_{y, m}$ on the diagonal respectively. The matrix $\boldsymbol{P}$ contains the information of the spatial dependency of the conductivity. The position dependent conductivity, that was introduced in (6), is described by a double Fourier series and its coefficients are arranged in the block-Toeplitz matrix $\boldsymbol{P}^{(14)}$. By this arrangement, multiplication of a vector with the block-Toeplitz matrix, results in a $2 \mathrm{D}$ convolution of the coefficients and thus a multiplication of the series in the spatial domain. The incorporation of the spatially dependent conductivity causes thereby a coupling of the harmonics. The Fourier representation of the conductivity in the conducting region for plate 2 (Fig. 2, which contains a slit pattern, is shown in Fig. 3. It is important to incorporate a sufficient number of harmonics to describe the conductivity distribution. If too few harmonics are used, the conductivity in the slits will not be equal to zero, which results in a less accurate result of the field computation. Especially for very thin slits, this means the number of harmonics that has to be used is relatively high.

Substituting (14) into (15) it is obtained that

$$
\frac{\partial^{2}}{\partial z^{2}}\left[\begin{array}{l}
\mathbf{h}_{\mathbf{x}} \\
\mathbf{h}_{\mathbf{y}}
\end{array}\right]=\mathbf{G F}\left[\begin{array}{l}
\mathbf{h}_{\mathbf{x}} \\
\mathbf{h}_{\mathbf{y}}
\end{array}\right]
$$

An eigenvalue decomposition is performed on the result of the matrix multiplication GF, to obtain the propagation information for the region with varying conductivity

$$
\left[\begin{array}{l}
\mathbf{Q}_{\mathbf{x}} \\
\mathbf{Q}_{\mathbf{y}}
\end{array}\right] \boldsymbol{\Lambda}^{2}\left[\begin{array}{l}
\mathbf{Q}_{\mathbf{x}} \\
\mathbf{Q}_{\mathbf{y}}
\end{array}\right]^{-1}=\mathbf{G F},
$$

where $\boldsymbol{\Lambda}$ is a diagonal matrix containing the vector $\lambda$ with propagation constants on the diagonal and $\mathbf{Q}_{\mathbf{x}}$ and $\mathbf{Q}_{\mathbf{y}}$ are

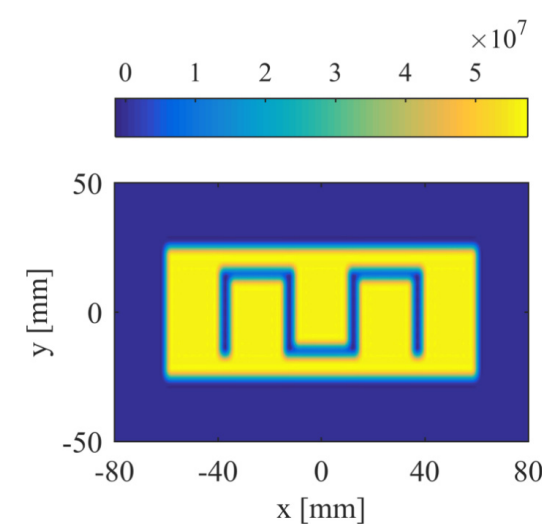

Fig. 3. Fourier representation of the conductivity in the conducting region, for $N=120$ and $M=42$

matrices containing the eigenvectors belonging to each eigenvalue. Because in (18) a double derivative to $z$ is performed, the matrix $\Lambda$ is squared in (19). Using the obtained propagation information, the $z$-dependent solution can be constructed. The solution consists, as is standard in the Fourier based model for a Cartesian coordinate system, of an upward traveling and downward traveling wave. The Fourier coefficients of the magnetic field strength components $\mathbf{h}_{\mathbf{x}}, \mathbf{h}_{\mathbf{y}}$ are then equal to

$$
\begin{aligned}
& \mathbf{h}_{\mathbf{x}}=\mathbf{Q}_{\mathbf{x}}\left(\mathbf{E}^{+}(\lambda, z) \mathbf{c}^{+}-\mathbf{E}^{-}(\lambda, z) \mathbf{c}^{-}\right) \\
& \mathbf{h}_{\mathbf{y}}=\mathbf{Q}_{\mathbf{y}}\left(\mathbf{E}^{+}(\lambda, z) \mathbf{c}^{+}-\mathbf{E}^{-}(\lambda, z) \mathbf{c}^{-}\right),
\end{aligned}
$$

where

$$
\begin{aligned}
& \mathbf{E}^{+}(\boldsymbol{\lambda}, z)=\operatorname{diag}\left(e^{\lambda z}\right) \\
& \mathbf{E}^{-}(\boldsymbol{\lambda}, z)=\operatorname{diag}\left(e^{-\lambda z}\right)
\end{aligned}
$$

The vectors $\mathbf{c}^{+}$and $\mathbf{c}^{-}$contain unknowns per harmonic pair. To obtain the unknowns of each region, boundary conditions are applied between the regions as also explained in ${ }^{(13)}$. This will form a system of linear equations which can be solved to determine all unknowns. The z-component of the magnetic field strength $\mathbf{h}_{\mathbf{z}}$ is determined using (3). Assuming a constant permeability throughout the region if follows that,

$$
H_{z}=-\int \frac{\delta H_{x}}{\delta x}+\frac{\delta H_{y}}{\delta y} d z
$$

which gives

$$
\begin{aligned}
\mathbf{h}_{\mathbf{z}}=-j & \left(\mathbf{K}_{\mathbf{x}} \mathbf{Q}_{\mathbf{x}}+\mathbf{K}_{\mathbf{y}} \mathbf{Q}_{\mathbf{y}}\right) \mathbf{\Lambda}^{-1} \\
& \left(\mathbf{E}^{+}(\boldsymbol{\lambda}, z) \mathbf{c}^{+}+\mathbf{E}^{-}(\boldsymbol{\lambda}, z) \mathbf{c}^{-}\right) .
\end{aligned}
$$

The coefficients of the magnetic flux density components can be obtained using (5). The solution for the induced current density components, $\mathbf{j}_{\mathbf{x}}^{\text {ind }}, \mathbf{j}_{\mathbf{y}}^{\text {ind }}$ and $\mathbf{j}_{\mathbf{z}}^{\text {ind }}$ are determined using (2). For the $x$-and $y$-components it is obtained that

$$
\begin{gathered}
\mathbf{j}_{\mathbf{x}}^{\text {ind }}=\mathbf{Q}_{\mathbf{y}} \Lambda\left(\mathbf{E}^{+}(\lambda, z) \mathbf{c}^{+}+\mathbf{E}^{-}(\lambda, z) \mathbf{c}^{-}\right)-j \mathbf{K}_{\mathbf{y}} \mathbf{h}_{\mathbf{z}} \\
\ldots \ldots \ldots \ldots \ldots \ldots \ldots \ldots \\
\mathbf{j}_{\mathbf{y}}^{\text {ind }}=-\mathbf{Q}_{\mathbf{x}} \boldsymbol{\Lambda}\left(\mathbf{E}^{+}(\lambda, z) \mathbf{c}^{+}+\mathbf{E}^{-}(\lambda, z) \mathbf{c}^{-}\right)+j \mathbf{K}_{\mathbf{x}} \mathbf{h}_{\mathbf{z}} .
\end{gathered}
$$

For the $z$-component it follows from (2) that 


$$
J_{z}=\frac{d H_{x}}{d y}-\frac{d H_{y}}{d x},
$$

and for the Fourier coefficients that

$$
\mathbf{j}_{\mathbf{z}}^{\text {ind }}=j \mathbf{K}_{\mathbf{y}} \mathbf{h}_{\mathbf{x}}-j \mathbf{K}_{\mathbf{x}} \mathbf{h}_{\mathbf{y}} \cdot
$$

The expressions for the magnetic field components in the non-conducting regions are described in ${ }^{(13)}$. In the source region, where the coil is present, a Fourier description of the current density distribution of the coil is needed as a source term. When comparing the model with FEM the current density description is based upon the geometrical properties of an ideal source coil. However, when the model is compared to measurements, a different approach is used. Instead of describing the geometry of the coil, the field of the coil, without any conducting plate on top, is measured. From this measurement, a backward transformation is performed to obtain the source terms. In this way, coil imperfections are already incorporated in the source terms which excludes this as a source of error between measurement and simulation.

\section{Experimental Setup}

The experimental setup is shown in Fig. 4. The race-track shaped coil is made of fine rectangular copper wire. Properties of the coil are given in Table 1. The pick-up coil, which is measuring the field, is attached to an H-bridge to accurately place it above the source coil. It has been made sure that the H-bridge does not influence the measurement, and no additional conducting materials are present near the setup. The pick-up coil is made of $30 \mu \mathrm{m}$ diameter copper wire, wound around a non-magnetic core with a diameter of $1.7 \mathrm{~mm}$. The field is measured at $1 \mathrm{~mm}$ above the plates on a grid of $120 \mathrm{~mm} \times 50 \mathrm{~mm}$ with a resolution of $1 \mathrm{~mm}$. The $z$-component of the magnetic flux density is calculated from the measured voltage, $V_{\text {meas }}$, using

$$
B_{z, \text { meas }}=\frac{V_{\text {meas }}}{T_{m c} A_{m c} \omega}, \cdots \ldots \ldots \ldots \ldots \ldots \ldots \ldots \ldots
$$

where $A_{m c}$ is the surface of the measurement coil $\left(2.3 \mathrm{~mm}^{2}\right)$ and $T_{m c}$ the number of turns of the measurement coil, which is equal to 103 and $\omega$ the radial frequency of the induced voltage. The three plates that are analyzed are shown in Fig. 2.

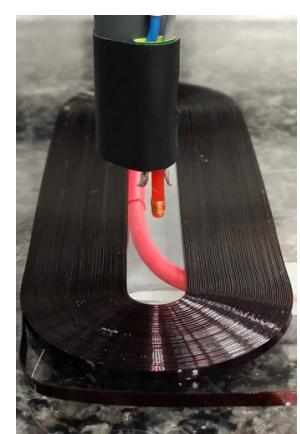

(a)

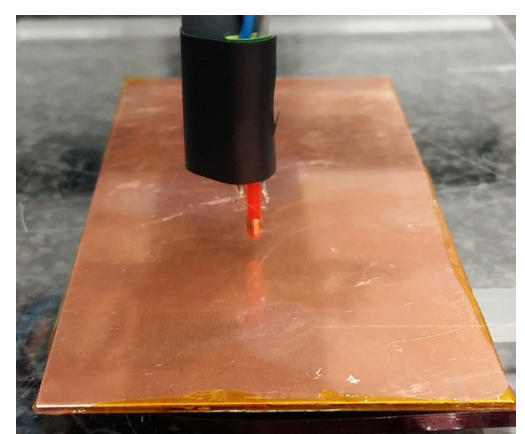

(b)
Fig. 4. The used measurement setup. a) Source coil (race-track shaped) with above it a very small pick-up coil, wound around a red non-magnetic core. b) A copper plate is placed on top of the coil and the pick-up coil is located above
The copper plates are placed directly on top of the coil, therefore, the bottom side of the plates is covered with a polyimide film for isolation. The conductivity of the plates during the measurement is assumed equal to $56 \mathrm{MS} / \mathrm{m}$.

\section{Validation of Harmonic Model}

To validate the developed harmonic model, it is applied to the electromagnetic configuration shown in Fig. 1. Properties of the configuration are given in Table 1 . The results obtained with the harmonic model are firstly compared to FEM. The $z$-component of the magnetic flux density, at $1 \mathrm{~mm}$ above the plates will be simulated. For the calculation of eddy currents and related fields with the developed model, $N$ and $M$ are set to 120 and 42 respectively. This is the maximum number of harmonics that can be included with the computational memory available (32.0 GB; MATLAB R2017a). The Fourier description of the conductivity description is close to zero inside the slits.

5.1 Validation using FEM To compare the harmonic model to FEM, the electromagnetic configuration of Fig. 1 is implemented in Flux 3D software ${ }^{(15)}$. The conductivity of the copper plates is considered to be $56 \mathrm{MS} / \mathrm{m}$ and the current through the source coil has a rms value of $3.2 \mathrm{~A}$ and the frequency is equal to $200 \mathrm{~Hz}$ or $2 \mathrm{kHz}$. In the $\mathrm{FE}$ model a second-order mesh is applied with a mesh-size of $0.5 \mathrm{~mm}$ in the conducting plate. At a frequency of $2 \mathrm{kHz}$, the skin depth of the copper is equal to $1.5 \mathrm{~mm}$. This means that 3 mesh elements are created per skin depth, which should lead to a proper calculation of the eddy current distribution with the FEM. In total, 506408 volumetric mesh elements are created in the FE model.

The simulated induced current densities in the several plates is depicted in Fig. 5. For plate 1 and 2, the conductivity is correctly incorporated and no current is flowing on the locations of the slits. For plate 3, however, a small current is flowing in the center slit and more harmonics should be added to get a more accurate current density description. Furthermore, it is clear that the slits reduce the overall current density, however, the peak current is increased.

In Fig. 6 the absolute value of the $z$-component of the magnetic flux density at $1 \mathrm{~mm}$ above plate 2 is shown. The result of the analytical (harmonic) model is depicted for a frequency of $200 \mathrm{~Hz}$ in Fig. 6(a) and for $2 \mathrm{kHz}$ in Fig. 6(c). The difference with the FEM is shown in Fig. 6(b) and Fig. 6(d). The root mean square (rms) error between the models is given in Table 2 for all three plates and both frequencies. At $200 \mathrm{~Hz}$, the reaction field due to the induced eddy currents is of very low influence on the magnetic field distribution. As a result, the rms error with FEM at $200 \mathrm{~Hz}$ is lower than at $2 \mathrm{kHz}$ for plates 2 and 3 .

The convergence of the harmonic model is depicted in Fig. 7. The rms error with respect to the result of the FEM has been calculated for a range of harmonic number pairs. To properly visualize the result, for each plate a different $y$-axis is created. The result of plate 1 seems to have converged to a final rms error as the result is fluctuating with approximately $0.04 \mathrm{mT}$ around an avarage value of $0.4 \mathrm{mT}$. The rate of decay of the rms difference is higher for plate 2 in comparison with plate 3 . Plate 3 has in comparison with the other two plates a relatively large rms difference with the FEM. The 


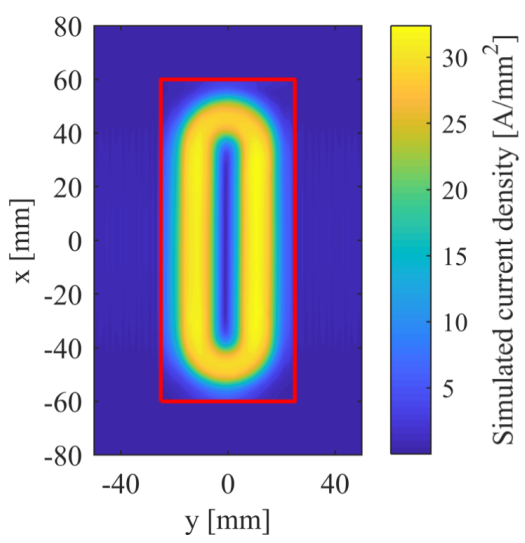

(a)

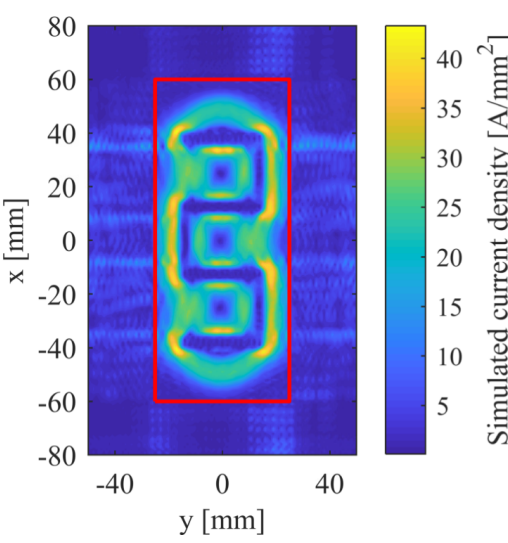

(b)

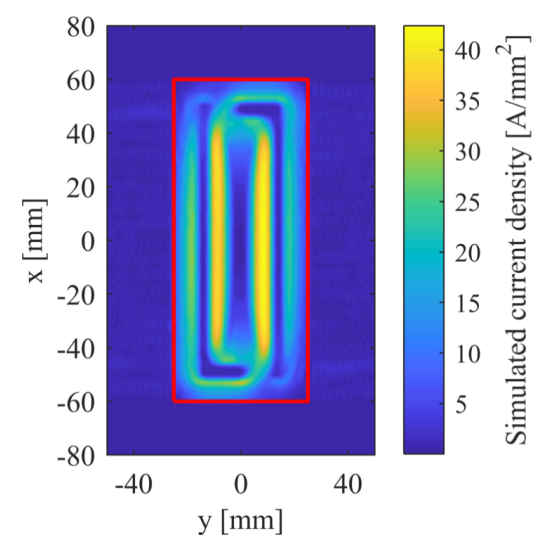

(c)

Fig. 5. Simulated current density in the conducting region (Region 4). The red square indicates the position of the plate. a) Plate 1. b) Plate 2. c) Plate 3

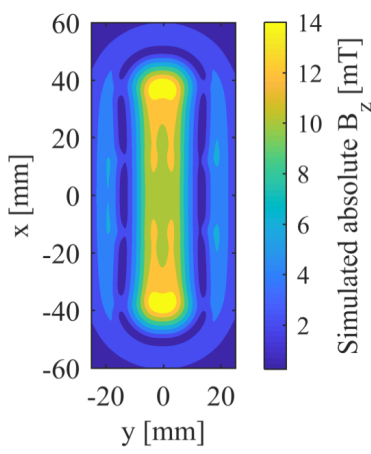

(a)

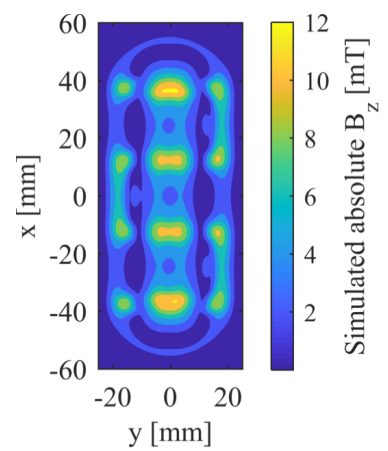

(c)

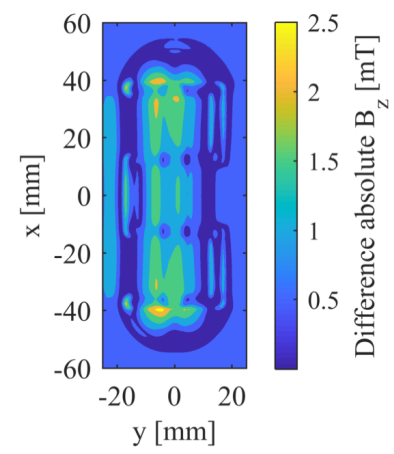

(b)

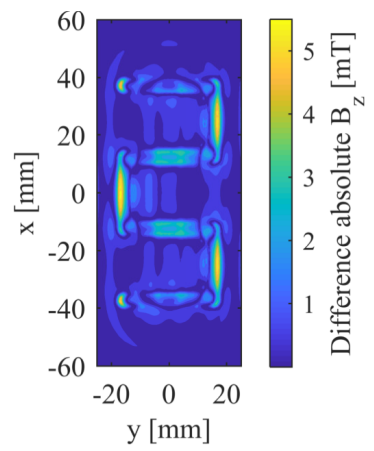

(d)
Fig. 6. Simulated absolute magnetic flux density in the $z$-direction $1 \mathrm{~mm}$ above plate 2 . a) Result from the harmonic model at $200 \mathrm{~Hz}$. b) Absolute difference with FEM at $200 \mathrm{~Hz}$. c) Result from the harmonic model at $2 \mathrm{kHz}$.

d) Absolute difference with FEM at $2 \mathrm{kHz}$

Table 2. rms error in absolute $B_{z}$ between the analytical model and FEM

\begin{tabular}{l|l|l} 
& $f=200 \mathrm{~Hz}$ & $f=2 \mathrm{kHz}$ \\
\hline \hline Plate 1 & $0.96 \mathrm{mT}$ & $0.38 \mathrm{mT}$ \\
Plate 2 & $0.94 \mathrm{mT}$ & $1.03 \mathrm{mT}$ \\
Plate 3 & $0.86 \mathrm{mT}$ & $2.77 \mathrm{mT}$
\end{tabular}

high spatial resolution in the $y$-direction of the slit pattern of plate 3 is causing the convergence of this plate to be relatively slow.

5.2 Validation using Measurements The measured magnetic flux densities at $2 \mathrm{kHz}$ with the different plates placed on top of the coil are shown in Fig. 8(a), Fig. 9(a) and Fig. 10(a). The absolute difference between the model

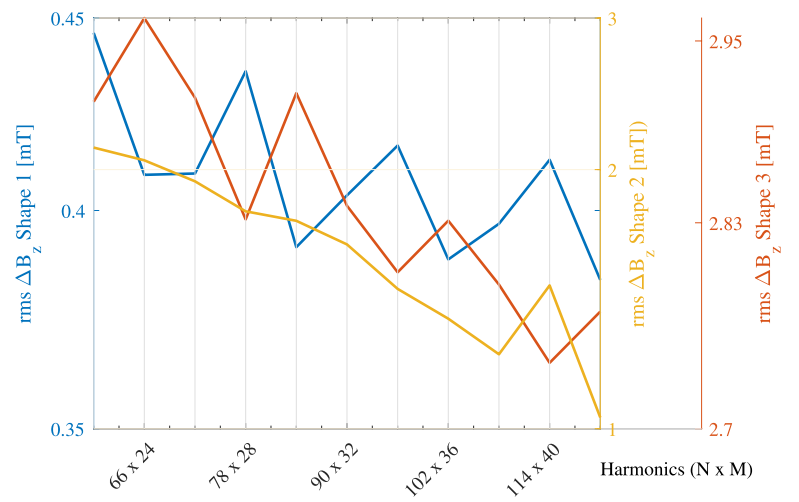

Fig. 7. Convergence of the rms error of the harmonic model with respect to the result of the FEM

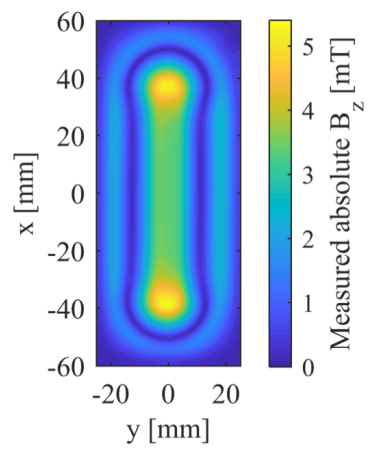

(a)

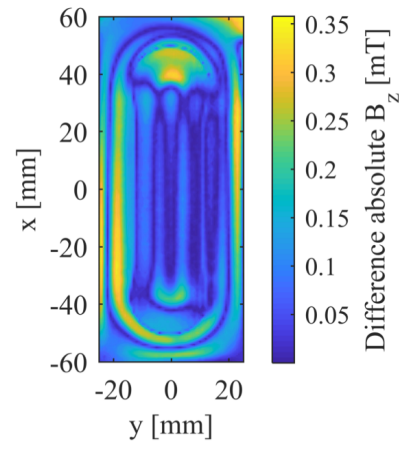

(b)
Fig. 8. Results for plate 1 at $2 \mathrm{kHz}$. a) Measured magnetic flux density in the $z$-direction. b) Absolute error with respect to the harmonic model

and the measurement is shown in Fig. 8(b), Fig. 9(b) and Fig. 10(b). The rms value of the error for the three plates is given in Table 3. It can be seen in Fig. 8, that for plate 1 the model and measurement are in good agreement, with an rms absolute error of $0.14 \mathrm{mT}$. For plate 2 and 3 the error is larger, $1.18 \mathrm{mT}$ and $1.57 \mathrm{mT}$ respectively. As there are no slit patterns in plate 1 , the used number of harmonics is relatively high. The errors in the fields above plate 2 and plate 3 are due to the truncation of the harmonics in both directions as was shown in the convergence analysis in the previous section. For higher frequencies, the eddy current distribution is concentrated around the slits. To model these distributions, the 


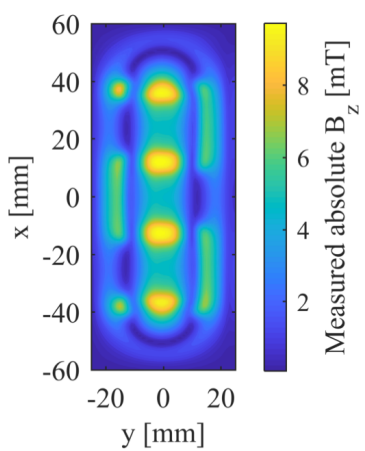

(a)

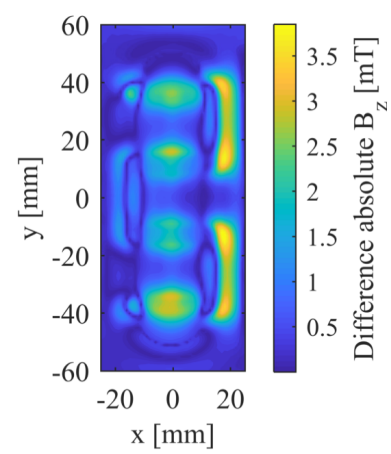

(b)
Fig. 9. Results for plate 2 at $2 \mathrm{kHz}$. a) Measured magnetic flux density in the $z$-direction. b) Absolute error with respect to the harmonic model

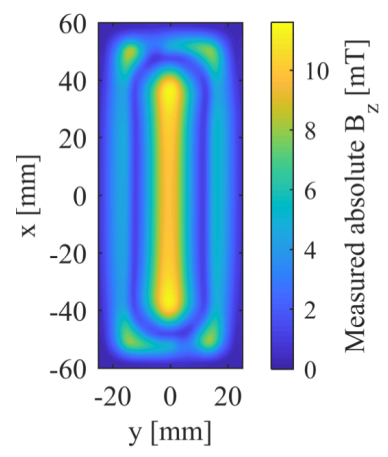

(a)

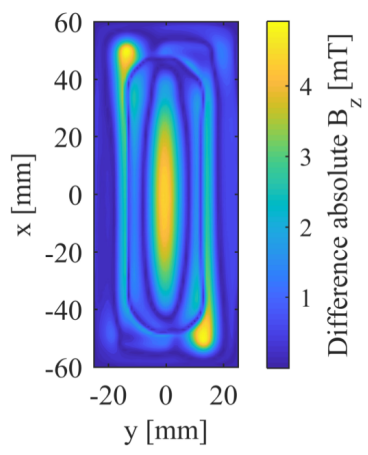

(b)
Fig. 10. Results for plate 3 at $2 \mathrm{kHz}$. a) Measured magnetic flux density in the $z$-direction. b) Absolute error with respect to the harmonic model

Table 3. rms error in absolute $B_{z}$ between the harmonic model and the measurement

$$
\begin{array}{l|l} 
& f=2 \mathrm{kHz} \\
\hline \hline \text { Plate 1 } & 0.14 \mathrm{mT} \\
\text { Plate 2 } & 1.18 \mathrm{mT} \\
\text { Plate 3 } & 1.57 \mathrm{mT}
\end{array}
$$

number of harmonics needs to be relatively high. Increasing the number of harmonics would, therefore, enhance the simulated magnetic flux density if a stable and smooth solution can be obtained.

\section{Conclusion}

The paper describes a semi-analytical modeling method, to model eddy currents in slitted conducting plates. The Fourier based solutions include a spatially varying conductivity in the solutions of the magnetic field and induced current density components. The model is compared to the finite element method and measurements. The field distribution, due to three different copper plates, of which two contained a slit pattern, placed on a source coil has been analyzed. At $200 \mathrm{~Hz}$, the rms error with respect to FEM is smaller than at $2 \mathrm{kHz}$, because the influence of the eddy currents is small at low frequencies. Furthermore, for higher frequencies the eddy currents are flowing near the slits, which requires a high number of harmonics to model. The convergence of the model, with respect to the FEM, shows that solution for the non-slitted plate has converged. Furthermore, the solution for plate 2 shows a higher convergence rate than plate
3. The modeled magnetic flux density due to plate 2 shows, at $2 \mathrm{kHz}$, an rms discrepancy of $0.38 \mathrm{mT}$ and $0.14 \mathrm{mT}$ with respect to FEM and the measurement, respectively. For the plate 3 , these errors are equal to $2.77 \mathrm{mT}$ and $1.57 \mathrm{mT}$, respectively. This error is caused by measurement uncertainties and by the limitation of the number of harmonics, which needs to be relatively high when the slit pattern has a high spatial resolution.

\section{References}

( 1 ) S.S. Nair, J. Wang, R. Chin, L. Chen, and T. Sun: "Analytical prediction of 3-d magnet eddy current losses in surface mounted pm machines accounting slotting effect", IEEE Transactions on Energy Conversion, Vol.32, No.2, pp.414-423 (2017)

( 2 ) M. Sawada, K. Hashimoto, Y. Kinoshita, M. Kuroda, T. Tamiya, Y. Shindo, and Y. Kawase: "Reduction in eddy current loss on rotor of spmsm driven by inverter", IEEJ Journal of IA, Vol.3, No.6, pp.428-436 (2014)

( 3 ) Y. Yoshida, K. Nakamura, O. Ichinokura, and K. Tajima: "Efficiency optimization of spm motor considering carrier harmonics based on electric and magnetic networks", IEEJ Journal of IA, Vol.3, No.6, pp.422-427 (2014)

( 4 ) R. Benlamine, F. Dubas, S.A. Randi, D. Lhotellier, and C. Espanet: "3-D numerical hybrid method for PM eddy-current losses calculation: Application to axial-flux pmsms", IEEE Trans. on Magnetics, Vol.51, No.7, pp.1-10 (2015)

( 5 ) T. Tera, H. Taki, and T. Shimizu: "Loss reduction of laminated core inductor used in on-board charger for evs", IEEJ Journal of IA, Vol.4, No.5, pp.626633 (2015)

( 6 ) J.M.M. Rovers, J.W. Jansen, and E.A. Lomonova: "Design and measurements of the double layer planar motor", in Electric Machines Drives Conference (IEMDC), 2013 IEEE International, pp.204-211 (2013)

( 7 ) L. Zhang, B. Kou, Y. Chen, and Y. Jin: "Analysis and calculation of eddy current braking force for an ironless linear synchronous motor with cooling system”, in 2016 19th International Conference on Electrical Machines and Systems (ICEMS), pp.1-5 (2016)

( 8 ) J.M.M. Rovers, J.W. Jansen, and E.A. Lomonova: "Disturbance effects of electrically conductive material in the air gap of a linear permanent magnet synchronous motor", IEEE Trans. on Magnetics, Vol.47, No.10, pp.26682671 (2011)

( 9 ) T. Nakano, Y. Kawase, T. Yamaguchi, M. Nakamura, and N. Nishikawa: "3$D$ finite element analysis of eddy current in laminated cores of the interior permanent-magnet motor", IEEE Trans. Magnetics, Vol.49, No.5, pp.19451948 (2013)

(10) A. Dwivedi, S.K. Singh, and R.K. Srivastava: "Analysis of permanent magnet brushless ac motor using Fourier transform approach", IET Electric Power Applications, Vol.10, No.6, pp.539-547 (2016)

(11) B. Hague: Electromagnetic Problems in Electrical Engineering, Oxford University Press, London, UK (1929)

(12) C.H.H.M. Custers, J.W. Jansen, and E.A. Lomonova: "2D semi-analytical modeling of eddy currents in multiple non-connected conducting elements", IEEE Trans. Magnetics, in press 2017, doi: 10.1109/TMAG.2017.2697946.

(13) J.P.C. Smeets, T.T. Overboom, J.W. Jansen, and E.A. Lomonova: "Threedimensional analytical modeling technique of electromagnetic fields of aircored coils surrounded by different ferromagnetic boundaries", IEEE Trans. on Magnetics, Vol.49, No.12, pp.5698-5708 (2013)

(14) S. Jayaraman, S. Esakkirajan, and T. Veerakumar: Digital Image Processing. McGraw-Hill Education (India) Pvt Limited (2011)

(15) Flux 12.2 User's guide, release 12.2 ed., Altair Engineering, Inc. (2016)

C.H.H.M. Custers (Non-member) was born in Weert, The Nether-

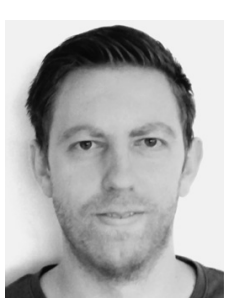
lands, in 1990. He received the M.Sc. degree in electrical engineering from the Eindhoven University of Technology (TU/e), Eindhoven, The Netherlands, in 2015. He is currently working towards the Ph.D. degree in the Electromechanics and Power Electronics Group of the Department of Electrical Engineering, Eindhoven University of Technology, Eindhoven, The Netherlands. His focus is on improving the accuracy of planar motors. 
J.W. Jansen (Non-member) received the M.Sc. degree (cum laude) in electrical engineering in 2003 and the Ph.D. degree in magnetically levitated planar actuator technology from the Eindhoven University of Technology, Eindhoven, The Netherlands. He then joined the Electromechanics and Power Electronics Group, Eindhoven University of Technology, where he is currently an Associate Professor. His research interests include magnetic levitation and the analysis and design of linear and planar actuators.
E.A. Lomonova (Non-member) was born in Moscow, Russia. She re-

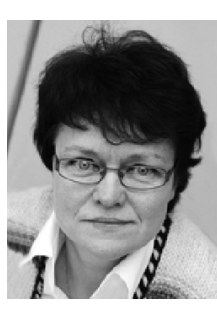

ics systems. ceived the M.Sc. (cum laude) and Ph.D. (cum laude) degrees in electromechanical engineering from the Moscow State Aviation Institute (TU), Moscow, in 1982 and 1993, respectively. She is currently a Full professor and Chair of the Electromechanics and Power Electronics group at the Eindhoven University of Technology, Eindhoven, The Netherlands. She has worked on electromechanical actuator design and optimization and development of advanced mechatron- 